\title{
NUMERICAL MODELING OF NON-BREAKING, IMPULSIVE BREAKING, AND BROKEN WAVE INTERACTION WITH ELEVATED COASTAL STRUCTURES
}

\author{
Hyoungsu Park, Oregon State University, Hyoungsu.Park@gmail.com \\ Trung Do, Colorado State University, tqdo@rams.colostate.edu \\ Tori Tomiczek, US Naval Academy, toritomi11@gmail.com \\ Daniel T. Cox, Oregon State University, dan.cox@oregonstate.edu \\ John W. van de Lindt, Colorado State University, jwv@engr.colostate.edu
}

\begin{abstract}
IMPORTANCE
Hurricanes generate elevated surge levels and strong waves that can cause extensive damage to buildings and other coastal infrastructure, especially those located in low-lying coastal regions. The history of recorded damage on buildings near the shoreline from past storms indicates that the intensity of storms and resulting damage has increased over the past 30 years (Emanuel, 2005). For example, the United States has been impacted by recent events such as Hurricanes Katrina (2005), Ike (2008), Sandy (2012), and Harvey (2017). Computational Fluid Dynamics (CFD) models have been widely developed and applied to estimate the wave pressure and forces; advances in recent years have been supported by an increase in computation power, which allows more detailed calculations of the complex hydrodynamics associated with wave action. The performance of CFD models must be validated or verified through detailed comparisons with benchmark tests (e.g. analytic solutions or physical experiments).
\end{abstract}

\section{MODEL VALIDATION AND COMPARISON}

Quantitative Computational Fluid Dynamics (CFD) model validation and inter-model comparisons between IHFOAM and ANSYS FLUENT were performed for pressures and forces on an elevated structure using a 1:10 physical model. Three types of wave breaking conditions (non-breaking, impulsive breaking, and broken) at the location of the structure were simulated numerically in IHFOAM (Fig. 1) and FLUENT (Fig. 2). The calculated time series of water surface elevation and horizontal and vertical pressures, and forces were compared with the measured data. To quantify variations between modeled and measured data as well as between the two CFD models, we introduce the impulse of residual and calculate the deviations for time series of forces and pressures on the structure (Fig. 3). The validation and comparison of results showed that the numerical models perform differently depending on the wave conditions even for the same CFD model set up. Non-breaking wave simulations showed the best agreement with experimental data for both FLUENT and IHFOAM, while broken wave trials showed the largest deviations. Results of this study indicate that future benchmarking tests for wave induced forces on an elevated structure must consider both horizontal and vertical forces due to various wave breaking conditions. The accuracy of simulated wave shoaling and breaking processes played a key role in precisely calculating the wave induced forces and pressures on the structure, but it was difficult for the CFD models to simulate the exact wave breaking conditions as the measurements. Finally, mesh sensitivity tests indicated that a finer mesh improves the performance of the simulations for broken wave conditions but generated minor changes for non-breaking wave conditions. Details of these model comparisons will be presented at the conference, with particular attention to trade-offs between computational cost and model accuracy.

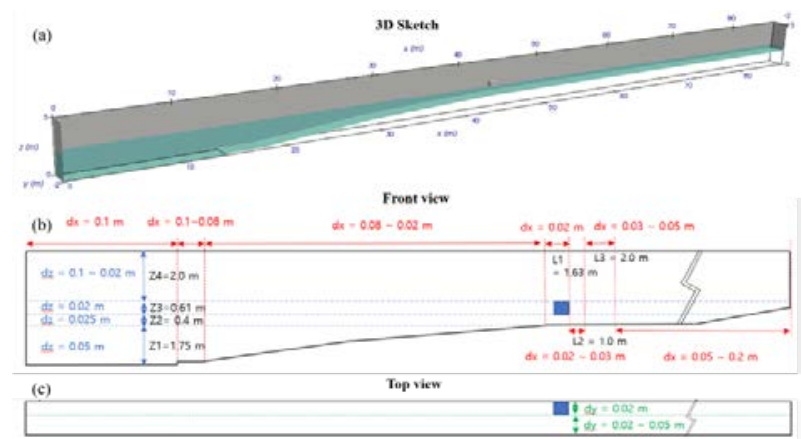

Fig.1: (a) Sketch of wave flume in the IHFOAM model, and the mesh configuration for (b) front view and (c) top view.

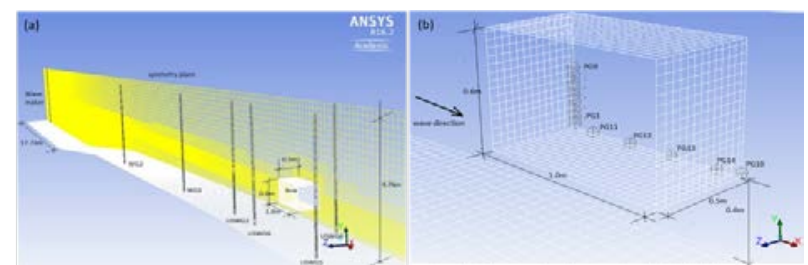

Fig 2: (a) The mesh sketch of wave flume (M2), and (b) instrument locations on the specimen for FLUENT.

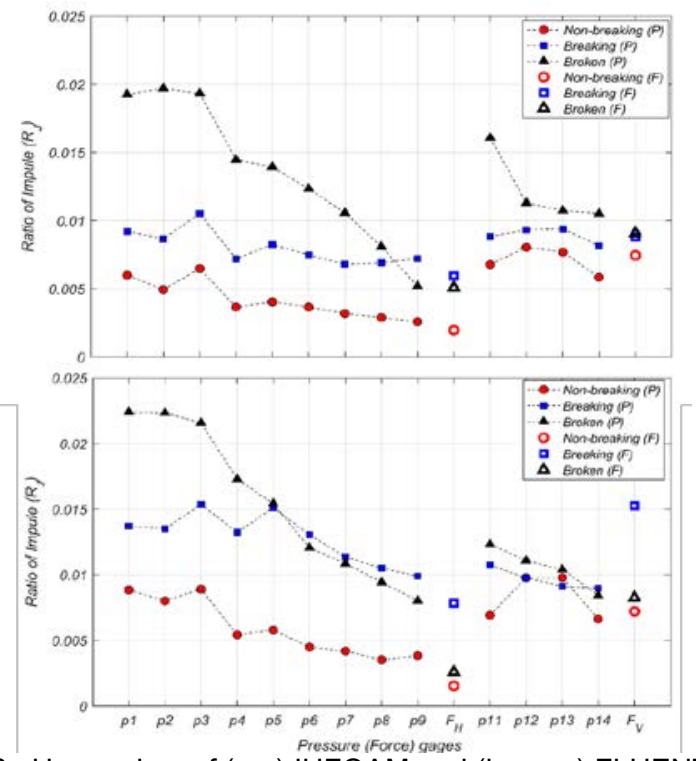

Fig 3: Uncertainty of (top) IHFOAM and (bottom) FLUENT model results for horizontal and vertical pressure and forces for non-breaking, breaking, and broken waves. 